CERN-TH.7252/94

\title{
DO THE PRESENT ELECTROWEAK PRECISION MEASUREMENTS LEAVE ROOM FOR EXTRA GENERATIONS?
}

\author{
V.A. Novikov*), \\ University of Guelph, Guelph, ON, N1G2W1, Canada \\ L.B. $\mathrm{Okun}^{*)}$ \\ Theoretical Physics Division, CERN \\ CH-1211 Geneva 23, Switzerland \\ A.N. Rozanov ${ }^{* *}$ \\ Particle Physics Experiments Division, CERN \\ CH-1211 Geneva 23, Switzerland \\ M.I. Vysotsky and V.P. Yurov \\ ITEP, Moscow 117259, Russia
}

\begin{abstract}
The recent data on $Z$ boson decays and on the $W$ mass are analyzed from the point of view of the possible existence of extra generations of leptons and quarks with masses $\gtrsim m_{Z} / 2$. Present experimental accuracy is not sufficient to exclude the existence of one extra generation of leptons and quarks, even with masses as low as $50 \mathrm{GeV}$.
\end{abstract}

*) Permanent address: ITEP, Moscow 117259, Russia.

**) On leave of absence from ITEP, Moscow 117259, Russia.

CERN-TH.7252/94

May 1994 
As is well known, the latest precision measurements of $Z$ and $W$ boson properties give, within the framework of the Minimal Standard Model (MSM), rather tight limits on the top quark mass, $m_{t}$, and the strong interaction coupling constant at the scale $m_{Z}, \alpha_{s}\left(m_{Z}^{2}\right)[1]$.

These limits follow from the comparison of the precision data with the theoretical formulae obtained in the approximation that takes into account, for each electroweak observable, the large Born term plus the rather small one-electroweak-loop term. It turns out that the loop corrections are especially small if (1) the Born approximation is based on $\bar{\alpha}$ (the value of the running electromagnetic coupling $\alpha\left(q^{2}\right)$ at $q^{2}=m_{Z}^{2}$ ), and (2) the value of the top-quark mass lies between 150 and $200 \mathrm{GeV}$ [2]. Thus the experimental data are essentially described by the $\bar{\alpha}$-Born approximation.

It is important to stress that this approximation is identical for a variety of models that extend the MSM by including new physics (supersymmetry, technicolour, new sequential generations, etc.). To a large extent, our $\bar{\alpha}$-Born approximation is model-independent. Of course, the loop corrections and hence the fitted values of $m_{t}$ and $\alpha_{s}\left(m_{Z}^{2}\right)$ would be different for different models, but for some of them the differences may be rather small.

In this paper we discuss the sensitivity of the electroweak experimental data to the existence of additional sequential generations of leptons and quarks which could be heavy enough not to be produced in the $Z$ decays, but at the same time might lie just above the current experimental bounds for their masses.

We also analyze the possibility that contributions of such relatively light fermions (which we refer to as the fourth generation) may be compensated by those of much heavier fermions (fifth generation). We find that such a compensation in all three "hadron-free" observables $m_{W} / m_{Z}, g_{A}$ and $g_{V} / g_{A}-$ is practically impossible.

Let us start from the simplest case, that of a degenerate fourth generation, where the masses of the up and down quarks and of the up and down leptons are equal:

$$
m_{U}=m_{D}=m_{Q}, \quad m_{N}=m_{E}=m_{L}
$$

In the subsequent equations, we introduce a variable $\ell$ :

$$
\ell=m_{Q}^{2} / m_{Z}^{2} \quad \text { for quarks }, \quad \ell=m_{L}^{2} / m_{Z}^{2} \quad \text { for leptons } .
$$

For the sum of the contributions of quarks and leptons we assume that $m_{Q}=m_{L}=m_{4}$ (see Figures 1 - 4).

New fermion generations affect the ratio $m_{W} / m_{Z}$ and the width and the decay asymmetries of the $Z$ boson through the $W$ and $Z$ self-energies which modify the functions $V_{m}, V_{A}, V_{R}$ (defined in Ref. [3]) which describe radiative corrections to $m_{W} / m_{Z}, g_{A}$ and $g_{V} / g_{A}$.

The latest experimental values (obtained at colliders) of the functions $V_{i}$ and their theoret- 
ically predicted fitted values within the framework of the MSM are:

$$
\begin{array}{ll}
V_{m}^{e x p}=1.84(1.29), & V_{m}^{M S M}=2.42(0.42)_{-0.18}^{+0.06} \\
V_{A}^{e x p}=1.85(0.77), & V_{A}^{M S M}=1.85(0.62)_{-0.46}^{+0.15} \\
V_{R}=-0.58(0.58), & V_{R}^{M S M}=-0.93(0.49)_{-0.23}^{+0.12}
\end{array}
$$

The experimental and theoretical one- $\sigma$ uncertainties are given in brackets, while the central theoretical values and their shifts correspond to

$$
m_{H}=300_{-240}^{+700} \mathrm{GeV} .
$$

The contribution from a fourth generation doublet would change the theoretical predictions:

$$
V_{m} \rightarrow V_{m}+\Delta^{4} V_{m}, \quad V_{A} \rightarrow V_{A}+\Delta^{4} V_{A}, \quad V_{R} \rightarrow V_{R}+\Delta^{4} V_{R} .
$$

The analytical expressions for $\Delta^{4} V_{i}$ for a quark or lepton doublet are given by (neglecting gluonic corrections):

$$
\begin{aligned}
\Delta^{4} V_{m} & =\frac{4}{9} N_{c}\left\{\left[(1-l) F(l)-\left(1-l / c^{2}\right) F\left(l / c^{2}\right)\right]+2 s^{2}\left[\left(1-l / c^{2}\right) F\left(l / c^{2}\right)-\right.\right. \\
- & \left.(1+2 l) F(l)]+4 s^{4}\left(Q_{U}^{2}+Q_{D}^{2}\right)\left[(1+2 l) F(l)-\frac{1}{3}\right]\right\} \\
\Delta^{4} V_{A} & =\frac{4}{9} N_{c}\left\{\left[1-l+\left(6 l^{2}-3 l\right) F(l)\right]+\left[4 s^{4}\left(Q_{U}^{2}+Q_{D}^{2}\right)-\right.\right. \\
& \left.\left.-2 s^{2}\right]\left[2 l+1-12 l^{2} F(l)\right]\right\} /(1-4 l), \\
\Delta^{4} V_{R}= & -\frac{4}{9} N_{c}\left\{3 l F(l)-4 s^{2} c^{2}\left(Q_{U}^{2}+Q_{D}^{2}\right)\left[(1+2 l) F(l)-\frac{1}{3}\right]\right\},
\end{aligned}
$$

where for quark doublet $N_{c}=3, Q_{U}=2 / 3, Q_{D}=-1 / 3$; for lepton doublet $N_{c}=1, Q_{U}=0$, $Q_{D}=-1$

$$
\begin{gathered}
s^{2} c^{2} \equiv \sin ^{2} \theta \cos ^{2} \theta=\frac{\pi \alpha\left(m_{Z}\right)}{\sqrt{2} G_{\mu} m_{Z}^{2}}, \\
s^{2}=0.2312(3), \quad c^{2}=1-s^{2} ; \\
F(l)= \begin{cases}2\left[1-\sqrt{4 l-1} \arcsin \frac{1}{\sqrt{4 l}}\right], & l>1 / 4 \\
2\left[1-\sqrt{1-4 l} \ln \frac{1+\sqrt{1-4 l}}{\sqrt{4 l}}\right], & l<1 / 4\end{cases} \\
\left.F(l)\right|_{l \rightarrow \infty} \rightarrow 1 / 6 l+1 / 60 l^{2} .
\end{gathered}
$$

In the limit of a very heavy fourth generation of leptons and quarks, $l \gg 1$, one has (see Figs. 1b, 2b, 3b):

$$
\Sigma \Delta^{4} V_{m} \rightarrow-\frac{16}{9} s^{2}, \quad \Sigma \Delta^{4} V_{R} \rightarrow-\frac{8}{9}, \quad \Sigma \Delta^{4} V_{A} \rightarrow 0
$$


where $\Sigma$ denotes sum over leptons and quarks with $m_{Q}=m_{L}=m_{4}$. Equations (7) reflect the well-known fact of the non-decoupling of the heavy degrees of freedom in electroweak theory.

For a relatively light fourth generation with production threshold near the $Z$-pole $\Delta V_{m}$ and $\Delta V_{R}$ are regular (their derivatives being singular), while $\Delta V_{A}$ becomes singular itself:

$$
\begin{gathered}
\left.\Sigma \Delta V_{A}\right|_{l \rightarrow \frac{1}{4}+0} \rightarrow-\frac{\pi}{\sqrt{4 l-1}}\left(\frac{2}{3}-\frac{8}{3} s^{2}+\frac{32}{9} s^{4}\right) \\
\left.\Sigma \Delta V_{A}\right|_{l \rightarrow \frac{1}{4}-0} \rightarrow \frac{16}{9}-\frac{128}{9} s^{2}+\frac{512}{27} s^{4} .
\end{gathered}
$$

This singularity is of the same nature as that of $V_{A}$ arising in the limit $m_{t} \rightarrow m_{Z} / 2$ and was discussed in [3]. It originates from the derivative of the $Z$ boson self-energy, $d \Sigma /\left.d q^{2}\right|_{q^{2}=m_{Z}^{2}}$, which comes from the loop insertion into the $Z$ boson wave function. Perturbation theory is valid as long as the term $\alpha \Delta V_{A} \sim \alpha \sqrt{m_{Z} /\left(2 m_{4}-m_{Z}\right)}$ is less than unity. This condition is violated for $\left|2 m_{4}-m_{Z}\right| \sim \alpha^{2} m_{Z}$ and it appears as if we could use our formulas for very small $\left|2 m_{4}-m_{Z}\right| \ll \Gamma_{Z}$, as $\Gamma_{Z} \sim \alpha m_{Z}$. In reality experimentalists at LEP determine the $Z$ boson total width from the shape of the $Z$ boson production cross-section in the range $m_{Z}-\Gamma_{Z}<E<m_{Z}+\Gamma_{Z}$. Partial $Z$ boson widths are determined through cross-sections at the $Z$ pole, which do not depend on $V_{A}$, and through the total width. For $m_{Z}-\Gamma_{Z}<2 m_{4}<m_{Z}+\Gamma_{Z}$ one should not expand $\Sigma\left(q^{2}\right)$ but compare the shape of $Z$ resonance with the Breit-Wigner formula as modified by the contribution of the fourth generation. We shall not proceed in this way here but instead confine ourselves to the case $m_{4}>\left(m_{Z}+\Gamma_{Z}\right) / 2 \simeq 47 \mathrm{GeV}$; for $m_{4}=47$ GeV one has $\Sigma \Delta^{4} V_{A} \simeq-3$, while $\Sigma \Delta^{4} V_{m}$ and $\Sigma \Delta^{4} V_{R}$ are much smaller:

$$
\left.\Sigma \Delta^{4} V_{m}\right|_{l=1 / 4} \simeq 0.3,\left.\quad \Sigma \Delta^{4} V_{R}\right|_{l=1 / 4} \simeq-0.4 .
$$

From Fig. 2a one can see that an extra doublet of leptons with $m_{4} \simeq 47 \mathrm{GeV}$ destroys the agreement between experimental and theoretical values of $V_{A}$ by more than $3 \sigma$. (The existence of such light quarks is excluded by direct searches [6].) As for $\Delta^{4} V_{m}$ and $\Delta^{4} V_{R}$ (see Figs. 1,3), they are smaller than the respective experimental uncertainties.

Taking into account experimental uncertainties, it is reasonable to anticipate that for large enough $l$ the whole set of experimental data could be fitted with a slightly higher $m_{t}$ than that resulting in the absence of extra generations (for a given $m_{H}$ value). For smaller $l$ the fit should get worse mainly because of the rapid increase of $\Delta V_{A}$. The question is at what $l$ the fit will start to be inadequate. For a single extra generation, our procedure is analogous to that presented in [4], using, however, all the updated experimental data presented at the 1994 La Thuile - Moriond conferences [5]. The Minimal Standard Model fit of the data (LEP, SLD, CDF, UA2, CHARM, CDHS, CCFR) yields:

$$
m_{t}=180(9) \mathrm{GeV}, \alpha_{s}\left(m_{Z}^{2}\right)=0.124(5), \chi^{2} / \text { d.o.f. }=23 / 11,
$$

assuming $m_{H}=300 \mathrm{GeV}$. The introduction of extra generations leads to an increase in $\chi^{2}$, e.g. for one extra generation with $m_{L}=m_{Q}=50 \mathrm{GeV}$

$$
m_{t}=199 \mathrm{GeV}, \alpha_{s}\left(m_{Z}^{2}\right)=0.130, \chi^{2} / \text { d.o.f. }=26 / 11 .
$$


It is interesting to consider to what extent a heavier and non-degenerate $\left(m_{T}>m_{B}\right)$ fifth generation may cancel the contribution of the degenerate $\left(m_{U}=m_{D}, m_{N}=m_{E}\right)$ fourth generation. If $m_{B} \gg m_{Z}$ and we keep terms $\mathrm{O}\left(m_{B}^{2} / m_{Z}^{2}\right)$ and $\mathrm{O}(1)$, then contributions of the fifth quark or lepton doublet may be written in the form

$$
\begin{gathered}
\Delta^{5} V_{m}=\Delta^{5} V_{A}-\frac{2}{9} N_{c}+\frac{4}{9} N_{c} s^{2}\left(1+2 Q_{B}\right) \ln (1+x)+ \\
+\frac{4}{9} N_{c}\left(s^{2}-c^{2}\right)\left[\frac{1}{3}-\frac{2}{x^{2}}(1+x)+\frac{3}{x^{3}}\left(x+\frac{2}{3}-\frac{x^{3}}{6}\right) \ln (1+x)\right] \\
\Delta^{5} V_{A}=\frac{N_{c}}{3} \frac{m_{B}^{2}}{m_{Z}^{2}}\left[2+x-2\left(1+\frac{1}{x}\right) \ln (1+x)\right] \\
\Delta^{5} V_{R}=\Delta^{5} V_{A}-\frac{2}{9} N_{c}+\frac{2}{9} N_{c}\left(1+2 Q_{B}\right) \ln (1+x)
\end{gathered}
$$

where $1+x=m_{T}^{2} / m_{B}^{2}$.

Equations (12)-(14) for the case of quarks agree with Eqs. (14) and (15) of Ref. [7].

Note that $N_{c}\left(1+2 Q_{B}\right)=+1$ for quarks and -1 for leptons. If we assume, for the sake of simplicity, that quarks and leptons have the same $m_{T}$ and $m_{B}$, we get for the sum of their contributions:

$$
\begin{gathered}
\Sigma \Delta^{5} V_{m}=\Sigma \Delta^{5} V_{A}-\frac{8}{9}+\frac{16}{9}\left(s^{2}-c^{2}\right) \times \\
{\left[\frac{1}{3}-\frac{2}{x^{2}}(1+x)+\frac{3}{x^{3}}\left(x+\frac{2}{3}-\frac{x^{3}}{6}\right) \ln (1+x)\right]} \\
\Sigma \Delta^{5} V_{A}=\frac{4}{3} \frac{m_{B}^{2}}{m_{Z}^{2}}\left[2+x-2\left(1+\frac{1}{x}\right) \ln (1+x)\right], \\
\Sigma \Delta^{5} V_{R}=\Sigma \Delta^{5} V_{A}-\frac{8}{9},
\end{gathered}
$$

Inspection of Figs. 4, based on Eqs. (12)-(17), reveals that the fifth generation cannot cancel the contribution of the fourth generation in all three $V_{i}$ 's.

In conclusion, one additional generation of heavy quarks and leptons with degenerate masses is not excluded by the present precise measurements though their existence would make $\chi^{2}$ worse.

This work was partly supported by RFFR grant 93-02-14431. One of us (L.O.) is grateful to the CERN Theoretical Physics Division for warm hospitality during his visit. 


\section{References}

[1] G.Altarelli, Talk at the 1993 Marseilles EPS-HEP Conference (1993).

[2] V.A.Novikov et al., Mod.Phys.Lett. A8 (1993) 2529; CERN preprint TH.7217/94 (1994).

[3] V.A.Novikov et al., Nucl.Phys. B397 (1993) 35.

[4] V.A.Novikov et al., Phys.Lett. B331 (1994) 433.

[5] P.Clarke, Y.K.Kim, B.Pietrzyk, P.Siegrist, and M.Woods, Talks at the 1994 Moriond Conference "Electroweak Interactions and Unified Theories".

[6] Review of Particle Properties, Particle Data Group, Phys.Rev. D45 (1992); VII, 159; II, 4; VI, 31; IX, 18.

[7] C. Froggatt, R.Moorhouse and I.Knowles, Phys.Rev. D45 (1992) 2471.

\section{Figure Captions}

Fig. 1 Contribution of the fourth-generation quarks $(q)$, leptons $(l)$ and leptons and quarks $(l+q)$ to $V_{m}$ for masses $m_{4}$ in the range (a) $30-50 \mathrm{GeV}$ and (b) $50-300 \mathrm{GeV}$. Note the changes in scales. The peak at $45 \mathrm{GeV}$ is due to the $Z$ threshold, the dip at $40 \mathrm{GeV}$ is due to the $W$ threshold.

Fig. 2 Contribution of the fourth generation to $V_{A}$. Same notations as in Fig. 1 .

Fig. 3 Contribution of the fourth generation to $V_{R}$. Same notations as in Fig. 1 .

Fig. 4 Contribution of non-degenerate "fifth generation" to $V_{m}, V_{A}, V_{R}$, with $m_{T}=400 \mathrm{GeV}$ for the "top" lepton or quark, and $m_{B}$, the mass of the "bottom" lepton or quark, varying from 200 to $400 \mathrm{GeV}$. 